\title{
Mathematical Modeling and Optimal Control Strategy for a Discrete Time Drug Consumption Model
}

\author{
Abderrahim Labzai $\mathbb{D}^{1}{ }^{1}$ Abdelfatah Kouidere $\mathbb{D D}^{1},{ }^{1}$ Bouchaib Khajji $\mathbb{D}^{1},{ }^{1}$ Omar Balatif $\mathbb{D}^{2}{ }^{2}$ \\ and Mostafa Rachik (iD) \\ ${ }^{1}$ Laboratory of Analysis, Modeling and Simulation, Department of Mathematics and Computer Science, \\ Faculty of Sciences Ben M'Sik Sidi Othman, Hassan II University, Casablanca, Morocco \\ ${ }^{2}$ Laboratory of Dynamical Systems, Department of Mathematics, Faculty of Sciences El Jadida, Chouaib Doukkali University, \\ El Jadida, Morocco
}

Correspondence should be addressed to Abderrahim Labzai; labzaiabdo1977@gmail.com

Received 17 December 2019; Accepted 22 June 2020; Published 6 August 2020

Academic Editor: Ricardo López-Ruiz

Copyright (c) 2020 Abderrahim Labzai et al. This is an open access article distributed under the Creative Commons Attribution License, which permits unrestricted use, distribution, and reproduction in any medium, provided the original work is properly cited.

\begin{abstract}
The aim of this paper is to study and investigate the optimal control strategy of a discrete mathematical model of drug consumption. The population that we are going to study is divided into six compartments: potential drug users, light drug users, heavy drug users, heavy drug users-dealers and providers, temporary quitters of drug consumption, and permanent quitters of drug consumption. Our objective is to find the best strategy to reduce the number of light drug users, heavy drug users, heavy drug users-dealers and providers, and temporary quitters of drug consumption. We use four control strategies which are awareness programs through media and education, preventing contact through security campaigns, treatment, and psychological support along with follow-up. Pontryagin's maximum principle in discrete time is used to characterize the optimal controls. The numerical simulation is carried out using MATLAB. Consequently, the obtained results confirm the effectiveness of the optimization strategy.
\end{abstract}

\section{Introduction}

Drug addiction is a serious problem that affects a large population of the world causing some people diseases, psychological disorders, and in many cases it leads to death. Despite the efforts made to decrease the consumption of drugs, there is an ongoing increase of the actual number of people who use drugs by 20 million people from 2015 to 2016 according to the World Drug Report in 2018 [1]. It is estimated that 271 million people worldwide, which represents approximately 5.6 percent of the global population aged 15-64 years, used drugs at least once during 2017. About 35 million of drugs users suffer from drug use disorders. As regards to the number of deaths, the Global Burden of Disease Study 2017 estimated that, globally, in 2017, there were 585,000 deaths and 42 million years of "healthy" life lost as a result of the use of drugs $[2,3]$. Drugs consumption can cause some treated and untreated diseases such as hepatitis $\mathrm{C}$, heart disorders, high blood pressure, increase in the proportion of toxins in the body, impaired sexual activity, chronic gastric infections, dyspepsia, and infections of the pancreas gland. There are also some psychological and mental damages induced by drug use, for example, negligence of appearance, excessive nervousness, tension, and irritation. In addition, the drug users are cut off from the family atmosphere and even from the whole society, and their relationship with their families and friends collapses. Traffic accidents prevail in roads in many cases due to drugs consumption.

In terms of the financial effects of the spread of drug use, drug money can have negative consequences on economy in the sense that it makes countries poorer, especially when drug-related revenues make up a large part of the total economy of any society or country. This type of economy is 
called "illicit economy" as it weakens the rule of law and facilitates corruption, which in turn strengthens the illicit drug sector [1].

In Morocco, drugs remain a source of worry for the people and government as it remains illegal and forbidden in the country. Some epidemiological studies previously carried out in Morocco state that drug use is increasing among young people and women. So, in order to come up with welldesigned surveys on drug use in schools, Morocco adopted the Mediterranean School Survey Project on Alcohol and Other Drugs (MedSPAD) [4], which is supported by the Pompidou Group of the Council of Europe. MedSPAD surveys were conducted in Morocco in two big cities (Rabat and Salé) following a pilot survey in 2003, before the first nationwide study was undertaken in 2009 and the second in 2013. MedSPAD aims were to determine the prevalence of substance use among 15- 17-year-old young people in Morocco to determine the age of onset of drug use and to learn about teenagers' knowledge, perspectives, and behaviors regarding drugs. The project worked on identifying some of the predictive factors of drug use to help in formulating strong policies for facilitating mental health support and drug prevention in Moroccan schools. The surveys carried out by MedSPAD found initial drug use starts from a young age, which is alarming and indicates that preventive and counseling programs need to be executed for very young students (elementary school), prior to onset. Therefore, the effective school prevention policies and community intervention programs (prevention, treatment, and rehabilitation) must be developed to find an end to this problem [4].

Mathematical modeling of drug consumption has been studied by many researchers [5-11]. In fact, most of those researchers were interested in the continuous time models described by the differential equations. Recently, more attention has been given to discrete time models (see, $[8,12-16]$ and the references cited therein). The rationale behind using discrete modeling can be summarized as follows. Firstly, the statistical data are collected at discrete moments (day, week, month, or year). So, it is more direct, more accurate and timely to describe the disease using discrete time models compared to continuous time models. Secondly, the use of discrete time models can avoid some mathematical complexities such as choosing a function space and regularity of the solution. Thirdly, the numerical simulations of continuous time models are obtained by the way of discretization $[8,12]$.

Based on the aforementioned reasons, we will develop a discrete time model in order to study the dynamics of the population that uses drug and we will introduce two classes of drug users distributed into a compartment of heavy drug consumers, who do not influence other individuals, and the compartment of heavy drug users-dealers and providers of drug, who do influence other individuals. Also, we add to our model an element which was not taken into consideration in most previous research studies, namely, a group of heavy drug users who transform to heavy drug users-dealers and providers of drug.

In addition, in order to find the best strategy to reduce the number of light drug users, heavy drug users, and temporary quitters of drugs, we will use four control strategies: awareness programs through media and education, preventing contact through security campaigns, treatment, and psychological support along with follow-up.

The paper is organized as follows. In Section 2, we present our discrete mathematical model that describes the dynamics of the population that uses drugs. In Section 3, we present the optimal control problem for the proposed model where we give some results concerning the existence of the optimal controls and we characterize these optimal controls using Pontryagin's Maximum Principle in discrete time. Numerical simulations are given in Section 4. Finally, the conclusion is given in Section 5 .

\section{A Mathematical Model}

In this section, we present a discrete $\mathbf{P}_{k} \mathbf{L}_{k} \mathbf{H}_{k}^{+} \mathbf{H}_{k}^{-} \mathbf{Q}_{k}^{t} \mathbf{Q}_{k}^{p}$ mathematical model of drug consumers. The population under investigation is divided into six compartments: individuals who are not yet drug users but interact with drug users $P_{k}$, light or occasional drug users $L_{k}$, heavy drug users who do not influence other individuals $H_{k}^{+}$, heavy drug users-dealers and providers who do influence other individuals $H_{k}^{-}$, individuals who temporarily quit drug consumption $Q_{k}^{t}$, and individuals who permanently quit drug consumption $Q_{k}^{p}$, respectively.

\subsection{Description of the Model}

(i) The compartment $\mathbf{P}$ represents the potential drug users whose age is over adolescence and adulthood and who do not use drugs but may use drugs subsequently due to interaction with drug users. It is assumed that potential drug users can acquire drug consumption behavior and can become light drug consumers through effective contact with occasional drug users in some social occasions such as weddings, graduation ceremonies, and week-end parties. In other words, it is assumed that the acquisition of a drug consumption behavior is analogous to acquiring disease infection. This compartment is increased by the recruitment of individuals at rate $\Lambda$ and it is decreased by the rates $\alpha_{1}\left(P_{k} L_{k} / N\right), \alpha_{2}\left(P_{k} H_{k}^{-} / N\right)$. Some people of this compartment leave at a constant death rate of $\mu$ due to the total natural death rate $\mu P_{k}$.

(ii) The compartment $\mathbf{L}$ contains light drug users who can control their consumption during some events and occasions or they use drugs in a way that is unapparent to their social environment. This category of light drug users does not face any problems or negative consequences; their friends or family do not complain about their drug intake. Light drug users neither think about drugs very often nor do they feel a need to using drugs. When using drugs, they are able to handle their drug consumption without experiencing a loss of control. Drugs do not dominate their thoughts and they do not need to set limits when they use drugs. They are not prone to 
extreme mood swings, fighting, or being violent, and their number is increased when they start using it with a rate $\alpha_{1}\left(P_{k} L_{k} / N\right)$. In this compartment, some other individuals will leave at the rates $\beta_{2}\left(L_{k} H_{k}^{-} / N\right), \beta_{1} L_{k}$, and $\mu L_{k}$. Here, $\beta_{1}$ and $\beta_{2}$ are the rates of light drug consumers who transform into heavy drug consumers and drug dealers and providers consecutively. This compartment is increased by $\theta Q_{k}^{t}$ (at the rate $\theta$ ) due to temporary quitters who revert back to using the drug.

(iii) The compartment $\mathbf{H}^{+}$encompasses heavy drug consumers who do not influence other individuals and who are suffering from addiction to drug consumption. When an individual becomes a heavy drug user, they face a great difficulty to control or set limits to their consumption. Their job, their family, social circle, and health are all endangered. Despite these negative consequences, the heavy drug consumers are unable to quit using drugs. The heavy drug consumers may begin to disclaim that they have a problem; this disclaim can make it even more difficult for the person to get help. This compartment is increased by the rate $\beta_{1}\left(L_{k} H_{k}^{+} / N\right)$ and is decreased by the rate $\gamma H_{k}^{+}$and the rate $\mu H_{k}^{+}$due to natural death.

(iv) The compartment $\mathbf{H}^{-}$includes heavy drug users, dealers, and providers of drugs who do influence other individuals. They work to make big profits through illegal ways, i.e., a drug dealer can be defined as a person who sells drugs, of any type or quantity, in an illegal manner. A dealer can be an occasional seller of drugs by selling small quantities to cover the costs of their own drug use, or they can be highly organized groups and businessmen within high-organized operations that run like a serious business. They are increased by the rate $\alpha_{2}\left(P_{k} H_{k}^{-} / N\right), \beta_{2}\left(L_{k} H_{k}^{-} / N\right)$ and decreased by the rate $\gamma H_{k}^{-}$and the rate $\mu H_{k}^{-}$due to natural death.

(v) The compartment $\mathbf{Q}^{t}$ is composed of the individuals who temporarily quit drugs and are increased at the rate $\gamma\left(1-\sigma_{1}\right) H_{k}^{+}, \lambda \sigma_{2} H_{k}^{-}$, where $\left(1-\sigma_{1}\right)$ is the fraction of drug users who temporarily quit using drug (at a rate $\gamma$ ). $\sigma_{1}$ is the remaining fraction of heavy drug users who permanently and temporarily quit using drug (at the rate $\gamma$ and $\lambda$ ). This compartment is decreased by the natural death $\mu Q_{k}^{t}$, and the rate $\theta Q_{k}^{t}$ represents the persons who return to be light drug users.

(vi) The compartment $\mathbf{Q}^{p}$ includes individuals who permanently quit drugs. This compartment is increased at the rate $\gamma \sigma_{1} H_{k}^{+}$and $\lambda\left(1-\sigma_{2}\right) H_{k}^{-}$, where $\left(1-\sigma_{2}\right)$ is the fraction of drug users who permanently quit using drugs (at a rate $\gamma$ ). $\sigma_{2}$ is the remaining fraction of heavy drug users who permanently and temporarily quit using drugs (at a rate $\gamma)$. Some people of this compartment will die at the rate $\mu Q_{k}^{p}$.
The variables $\mathbf{P}_{k}, \mathbf{L}_{k}, \mathbf{H}_{k}^{+}, \mathbf{H}_{k}^{-}, \mathbf{Q}_{k}^{t}$, and $\mathbf{Q}_{k}^{p}$ are the numbers of the individuals in the six classes at time $k$, respectively. The unit $k$ can correspond to periods, phases, or years. It depends on the frequency of the survey studies as needed.

The following diagram will demonstrate the flow directions of individuals among the compartments. These directions are going to be represented by directed arrows (see Figure 1).

The total population size at time $k$ is denoted by $\mathbf{N}_{k}$ with $\mathbf{N}_{k}=\mathbf{P}_{k}+\mathbf{L}_{k}+\mathbf{H}_{k}^{+}+\mathbf{H}_{k}^{-}+\mathbf{Q}_{k}^{t}+\mathbf{Q}_{k}^{p}$.

2.2. Model Equations. By the addition of the rates at which individuals enter the compartment and also by subtracting the rates at which people leave the compartment, we obtain a difference equation for the rate at which the individuals of each compartment change over discrete time. Hence, we present the drug users infection model by the following system of difference equations:

$$
\left\{\begin{array}{l}
P_{k+1}=\Lambda+(1-\mu) P_{k}-\alpha_{1} \frac{P_{k} L_{k}}{N}-\alpha_{2} \frac{P_{k} H_{k}^{-}}{N}, \\
L_{k+1}=\left(1-\mu-\beta_{1}\right) L_{k}+\theta Q_{k}^{t}+\alpha_{1} \frac{P_{k} L_{k}}{N}-\beta_{2} \frac{L_{k} H_{k}^{-}}{N}, \\
H_{k+1}^{+}=\left(1-\mu-\beta_{3}-\gamma\right) H_{k}^{+}+\beta_{1} L_{k}+\beta_{2} \frac{L_{k} H_{k}^{-}}{N}+\alpha_{2} \frac{P_{k} H_{k}^{-}}{N}, \\
H_{k+1}^{-}=(1-\mu-\lambda) H_{k}^{-}+\beta_{3} H_{k}^{+}, \\
Q_{k+1}^{t}=(1-\mu-\theta) Q_{k}^{t}+\gamma\left(1-\sigma_{1}\right) H_{k}^{+}+\lambda \sigma_{2} H_{k}^{-}, \\
Q_{k+1}^{p}=(1-\mu) Q_{k}^{p}+\gamma \sigma_{1} H_{k}^{+}+\lambda\left(1-\sigma_{2}\right) H_{k}^{-},
\end{array}\right.
$$

where $P_{0} \geq 0, L_{0} \geq 0, H_{0}^{+} \geq 0, H_{0}^{-} \geq 0, Q_{0}^{t} \geq 0$, and $Q_{0}^{p} \geq 0$ are the given initial states.

\section{The Optimal Control Problem}

The strategies of control that we adopt consist of an awareness program through media and education, contact prevention through security campaigns, treatment, and psychological support along with follow-up. Our main goal in adopting these strategies is to minimize the number of light drug consumers, heavy drug consumers, heavy drug users-dealers, and the temporary quitters of drugs during the time steps $k=0$ to $T-1$ and also minimize the cost spent in applying these strategies. In this model, we include the four controls $u_{1, k}, u_{2, k}, u_{3, k}$, and $u_{4, k}$ that represent consecutively awareness programs through media and education, contact prevention through security campaigns, treatment, and psychological support along with follow-up as measures at time $k$. So, the controlled mathematical system is given by the following system of difference equations: 


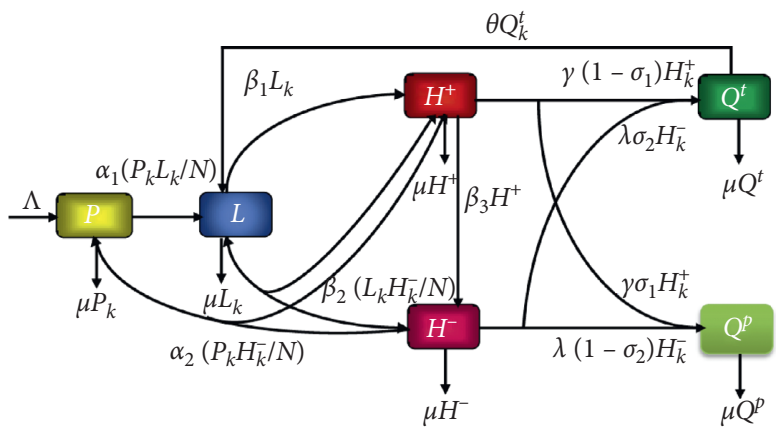

Figure 1: The flow between the five compartments $\mathbf{P} \mathbf{L} \mathbf{H}^{+} \mathbf{H}^{-} \mathbf{Q}^{t} \mathbf{Q}^{p}$.

$$
\left\{\begin{array}{l}
P_{k+1}=\Lambda+(1-\mu) P_{k}-\alpha_{1}\left(1-u_{1, k}\right) \frac{P_{k} L_{k}}{N}-\alpha_{2}\left(1-u_{2, k}\right) \frac{P_{k} H_{k}^{-}}{N}, \\
L_{k+1}=\left(1-\mu-\beta_{1}\right) L_{k}+\theta Q_{k}^{t}+\alpha_{1}\left(1-u_{1, k}\right) \frac{P_{k} L_{k}}{N}-\beta_{2}\left(1-u_{2, k}\right) \frac{L_{k} H_{k}^{-}}{N}, \\
H_{k+1}^{+}=\left(1-\mu-\beta_{3}-\gamma\right) H_{k}^{+}+\beta_{1} \frac{L_{k} H_{k}^{+}}{N}+\beta_{2}\left(1-u_{2, k}\right) \frac{L_{k} H_{k}^{-}}{N}+\alpha_{2}\left(1-v_{k}\right) \frac{P_{k} H_{k}^{-}}{N}-u_{3, k} H_{k}^{+}, \\
H_{k+1}^{-}=(1-\mu-\lambda) H_{k}^{-}+\beta_{3} H_{k}^{+}, \\
Q_{k+1}^{t}=(1-\mu-\theta) Q_{k}^{t}+\gamma\left(1-\sigma_{1}\right) H_{k}^{+}+\lambda \sigma_{2} H_{k}^{-}-u_{4, k} Q_{k}^{t}, \\
Q_{k+1}^{p}=(1-\mu) Q_{k}^{p}+\gamma \sigma_{1} H_{k}^{+}+\lambda\left(1-\sigma_{2}\right) H_{k}^{-}+u_{3, k} H_{k}^{+}+u_{4, k} Q_{k}^{t},
\end{array}\right.
$$

where $P_{0} \geq 0, L_{0} \geq 0, H_{0}^{+} \geq 0, H_{0}^{-} \geq 0, Q_{0}^{t} \geq 0$, and $Q_{0}^{p} \geq 0$ are the given initial states.

There are four controls $u_{1, k}=\left(u_{1,0}, u_{1,1}, \ldots, u_{1, T-1}\right)$, $u_{2, k}=\left(u_{2,0}, u_{2,1}, \ldots, u_{2, T-1}\right), \quad u_{3, k}=\left(u_{3,0}, u_{3,1}, \ldots, u_{3, T-1}\right)$, and $u_{4, k}=\left(u_{4,0}, u_{4,1}, \ldots, u_{4, T-1}\right)$. The first control can be interpreted as the proportion to be adopted to awareness programs through media and education. So, we note that $\left(1-u_{1, k}\right)\left(P_{k} L_{k} / N\right)$ is the proportion of the potential drug users who are protected from contacting light drug consumers at time step $k$. The second control can be interpreted as the proportion of contact prevention through security campaigns. We observe that $\left(1-u_{2, k}\right)\left(L_{k} H_{k}^{-} / N\right)$ is the proportion of the individuals who will be prevented to move from the class of light drug consumers towards the class of heavy drug users, dealers, and providers at time step $k$. The third control can be interpreted as the proportion of individuals to be subjected to treatment. So, we note that $u_{3, k} H_{k}^{+}$is the proportion of the individuals who will move from the class of heavy drug consumers towards the class of the individuals who permanently quit using drug at time step $k$. The fourth control can be interpreted as the proportion of individuals who will get psychological support along with follow-up. So, we observe that $u_{4, k} Q_{k}^{t}$ is the proportion of the individuals who temporarily quit using drugs and who will transform into the individuals who permanently quit drug at time step $k$.
The challenge that we face here is how to minimize the objective functional:

$$
\begin{aligned}
J\left(u_{1, k}, u_{2, k}, u_{3, k}, u_{4, k}\right)= & A_{T} L_{T}+B_{T} H_{T}^{+}+C_{T} H_{T}^{-}+D_{T} Q_{T}^{t} \\
& +\sum_{k=0}^{T-1}\left(A_{k} L_{k}+B_{k} H_{k}^{+}+C_{k} H_{k}^{-}+D_{k} Q_{k}^{t}\right) \\
& +\sum_{k=0}^{T-1} \frac{E_{k}}{2} u_{1, k}^{2}+\frac{F_{k}}{2} u_{2, k}^{2}+\frac{G_{k}}{2} u_{3, k}^{2}+\frac{M_{k}}{2} u_{4, k}^{2},
\end{aligned}
$$

where the parameters $A_{k}>0, B_{k}>0, C_{k}>0, D_{k}>0, E_{k}>0$, $F_{k}>0$, and $M_{k}>0$ are the cost coefficients, and they are selected to weigh the relative importance of $L_{k}, H_{k}^{+}, H_{k}^{-}, Q_{k}^{t}$, $u_{1, k}, u_{2, k}, u_{3, k}$, and $u_{4, k}$ at time $k . T$ is the final time.

In other words, we seek the optimal controls $u_{1, k}, u_{2, k}$, $u_{3, k}$, and $u_{4, k}$ such that

$$
J\left(u_{1, k}^{*}, u_{2, k}^{*}, u_{3, k}^{*}, u_{4, k}^{*}\right)=\min _{\left(u_{1}, u_{2}, u_{3}, u_{4}\right) \in U_{a d}^{4}} J\left(u_{1, k}, u_{2, k}, u_{3, k}, u_{4, k}\right),
$$

where $U_{a d}$ is the set of admissible controls defined by 


$$
\begin{aligned}
U_{a d}= & \left\{u_{i, k}=\left(u_{i, 0}, u_{i, 1}, \ldots, u_{i, T-1}\right),\right. \\
& \text { for } \left.i=1,2,3,4: a_{i} \leq u_{i, k} \leq b_{i} ; k=0,1,2, \ldots, T-1\right\} .
\end{aligned}
$$

The sufficient condition for the existence of the optimal controls $\left(u_{1}, u_{2}, u_{3}, u_{4}\right)$ for problems (2) and (3) comes from the following theorem.

Theorem 1. There exists the optimal controls $\left(u_{1, k}^{*}, u_{2, k}^{*}, u_{3, k}^{*}, u_{4, k}^{*}\right)$ such that

$$
J\left(u_{1, k}^{*}, u_{2, k}^{*}, u_{3, k}^{*}, u_{4, k}^{*}\right)=\min _{\left(u_{1}, u_{2}, u_{3}, u_{4}\right) \in U_{a d}^{4}} J\left(u_{1}, u_{2}, u_{3}, u_{4}\right),
$$

subject to the control system (2) with initial conditions.

Proof. Since the coefficients of the state equations are bounded and there is a finite number of time steps, $P=$ $\left(P_{0}, P_{1}, \ldots, P_{T}\right), L=\left(L_{0}, L_{1}, \ldots, L_{T}\right), H^{+}=\left(H_{0}^{+}, H_{1}^{+}, \ldots\right.$, $\left.H_{T}^{+}\right), H^{-}=\left(H_{0}^{-}, H_{1}^{-}, \ldots, H_{T}^{-}\right), Q^{t}=\left(Q_{0}^{t}, Q_{1}^{t}, \ldots, Q_{T}^{t}\right)$, and $Q^{p}=\left(Q_{0}^{p}, Q_{1}^{p}, \ldots, Q_{T}^{p}\right)$ are uniformly bounded for all $\left(u_{1}, u_{2}, u_{3}, u_{4}\right)$ in the control set $U_{a d}$; thus, $J\left(u_{1}, u_{2}, u_{3}, u_{4}\right)$ is bounded for all $\left(u_{1}, u_{2}, u_{3}, u_{4}\right) \in U_{a d}^{4}$. Since $J\left(u_{1}, u_{2}, u_{3}, u_{4}\right)$ is bounded, $\inf _{\left(u_{1}, u_{2}, u_{3}, u_{4}\right) \in U_{a d}^{4}} J\left(u_{1}, u_{2}, u_{3}, u_{4}\right)$ is finite, and there exists a sequence $\left(u_{1}^{j} ; u_{2}^{j} ; u_{3}^{j} ; u_{4}^{j}\right) \in U^{4}$ ad such that $\lim _{j \longrightarrow+\infty} J\left(u_{1}^{j} ; u_{2}^{j} ; u_{3}^{j} ; u_{4}^{j}\right)=\inf _{\left(u_{1}, u_{2}, u_{3}, u_{4}\right) \in U_{a d}^{4}} J\left(u_{1}, u_{2}, u_{3}, u_{4}\right)$ and corresponding sequences of states $P^{j}, L^{j}, H^{+j}, H^{-j}, Q^{t j}$, and $Q^{p j}$. Since there is a finite number of uniformly bounded sequences, there exist $\left(u_{1}^{*}, u_{2}^{*}, u_{3}^{*}, u_{4}^{*}\right) \in U_{a d}^{4}$ and $P^{*}, L^{*}, H^{+*}$, $H^{-*}, Q^{t *}$, and $Q^{p *} \in I R^{T+1}$ such that on a subsequence, $\left(u_{1}^{j} ; u_{2}^{j} ; u_{3}^{j} ; u_{4}^{j}\right) \longrightarrow\left(u_{1}^{*}, u_{2}^{*}, u_{3}^{*}, u_{4}^{*}\right), P^{j} \longrightarrow P^{*}, L^{j} \longrightarrow L^{*}$, $H^{+j} \longrightarrow H^{+j *}, H^{-j} \longrightarrow H^{-j *}, Q^{t j} \longrightarrow Q^{t *}$, and $Q^{p j} \longrightarrow$ $Q^{p *}$. Finally, due to the finite dimensional structure of system (2) and the objective function $J\left(u_{1}, u_{2}, u_{3}, u_{4}\right)$, $\left(u_{1}^{*}, u_{2}^{*}, u_{3}^{*}, u_{4}^{*}\right)$ is an optimal control with corresponding states $P^{*}, L^{*}, H^{+j *}, H^{-j *}$, and $Q^{p *}$. Therefore,

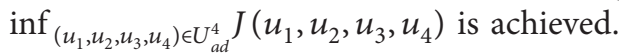

We apply the discrete version of Pontryagin's Maximum Principle [10, 14, 16-19]. The key idea is introducing the adjoint function to attach the system of difference equations to the objective functional resulting in the formation of a function called the Hamiltonian. This principle converts the problem of finding the control to optimize the objective functional subject to the state difference equation with initial condition to find the control to optimize Hamiltonian pointwise (with respect to the control).

We have the Hamiltonian $H_{k}$ at time step $k$, defined by

$$
H_{k}=A_{k} L_{k}+B_{k} H_{k}^{+}+C_{k} H_{k}^{-}+D_{k} Q_{k}^{t}+\frac{E_{k}}{2} u_{1, k}^{2}+\frac{F_{k}}{2} u_{2, k}^{2}
$$

$$
+\frac{G_{k}}{2} u_{3, k}^{2}+\frac{M_{k}}{2} u_{4, k}^{2}+\sum_{i=1}^{6} \zeta_{i, k+1} f_{i, k+1}
$$

where $f_{i, k+1}$ is the right side of the system of difference (2) of the $i$ th state variable at time step $k+1$.

Theorem 2. Given the optimal controls $\left(u_{1, k}^{*}, u_{2, k}^{*}\right.$, $\left.u_{3, k}^{*}, u_{4, k}^{*}\right) \in U_{\text {ad }}^{4}$ and the solutions $P^{*}, L^{*}, H^{+j *}, H^{-j *}, Q^{t *}$, and $Q^{p *}$ of the corresponding state system (2), there exist adjoint functions $\zeta_{1, k}, \zeta_{2, k}, \zeta_{3, k}, \zeta_{4, k}, \zeta_{5, k}$, and $\zeta_{6, k}$ satisfying

$$
\begin{aligned}
\zeta_{1, k}= & \zeta_{1, k+1}(1-\mu)+\alpha_{1}\left(1-u_{1, k}\right) \frac{L_{k}}{N}\left(\zeta_{2, k+1}-\zeta_{1, k+1}\right), \\
\zeta_{2, k}= & A_{k}+\alpha_{1}\left(\zeta_{2, k+1}-\zeta_{1, k+1}\right) \frac{P_{k}}{N}+\beta_{1}\left(\zeta_{3, k+1}-\zeta_{2, k+1}\right) \\
& +\alpha_{2}\left(1-u_{2, k}\right) \frac{H_{k}^{-}}{N}\left(\zeta_{3, k+1}-\zeta_{1, k+1}\right), \\
\zeta_{3, k}= & B_{k}+\zeta_{3, k+1}\left(1-\mu-\beta_{3}-\gamma\right)+\zeta_{4, k+1} \beta_{3}+\zeta_{5, k+1} \gamma\left(1-\sigma_{1}\right)+\zeta_{6, k+1} \gamma \sigma_{1} \\
& +\beta_{2}\left(1-u_{2, k}\right)\left(\zeta_{3, k+1}-\zeta_{2, k+1}\right) \frac{H_{k}^{-}}{N}+\zeta_{2, k+1}(1-\mu), \\
\zeta_{4, k}= & C_{k}+\alpha_{2}\left(1-u_{2, k}\right)\left(\zeta_{3, k+1}-\zeta_{1, k+1}\right) \frac{P_{k}}{N}+\beta_{2}\left(1-u_{2, k}\right)\left(\zeta_{3, k+1}-\zeta_{2, k+1}\right) \frac{L_{k}}{N}, \\
\zeta_{5, k}= & D_{k}+\zeta_{2, k+1} \theta+\zeta_{5, k+1}\left(1-\mu-\theta-u_{4, k}\right)+\zeta_{6, k+1} u_{4, k}-\zeta_{3, k+1} u_{3, k} \\
& +\zeta_{4, k+1}(1-\mu-\lambda)+\zeta_{5, k+1} \lambda \sigma_{2}+\zeta_{6, k+1}\left(\lambda\left(1-\sigma_{2}\right)+u_{3, k}\right), \\
\zeta_{6, k}= & \zeta_{6, k+1}(1-\mu) .
\end{aligned}
$$

With the transversality conditions at time T. $\zeta_{1, T}=\zeta_{6, T}=$ $0, \zeta_{2, T}=A_{T}, \zeta_{3, T}=B_{T}, \zeta_{4, T}=C_{T}$, and $\zeta_{5, T}=D_{T}$.

Furthermore, for $k=0,1,2, \ldots, T-1$, the optimal controls $u_{1, k}^{*}, u_{2, k}^{*}, u_{3, k}^{*}$, and $u_{4, k}^{*}$ are given by 


$$
\begin{aligned}
& u_{1, k}^{*}=\min \left[b ; \max \left(a, \frac{1}{E_{k}}\left[\left(\zeta_{2, k+1}-\zeta_{1, k+1}\right) \alpha_{1} \frac{P_{k} L_{k}}{N}\right]\right)\right], \\
& u_{2, k}^{*}=\min \left[d ; \max \left(c, \frac{1}{N F_{k}}\left[\alpha_{2} P_{k} H_{k}^{-}\left(\zeta_{3, k+1}-\zeta_{1, k+1}\right)+\left(\beta_{2} L_{k} H_{k}^{-}\right)\left(\zeta_{3, k+1}-\zeta_{2, k+1}\right)\right]\right)\right], \\
& u_{3, k}^{*}=\min \left[f ; \max \left(e, \frac{1}{G_{k}}\left[\left(\zeta_{3, k+1}-\zeta_{6, k+1}\right) H_{k}^{-}\right]\right)\right], \\
& u_{4, k}^{*}=\min \left[h ; \max \left(g, \frac{1}{M_{k}}\left[\left(\zeta_{5, k+1}-\zeta_{6, k+1}\right) Q_{k}^{t}\right]\right)\right] .
\end{aligned}
$$

Proof. The Hamiltonian at time step $k$ is given by

$$
\begin{aligned}
H_{k}= & A_{k} L_{k}+B_{k} H_{k}^{+}+C_{k} H_{k}^{-}+D_{k} Q_{k}^{t}+\frac{E_{k}}{2} u_{1, k}^{2}+\frac{F_{k}}{2} u_{2, k}^{2}+\frac{G_{k}}{2} u_{3, k}^{2}+\frac{M_{k}}{2} u_{4, k}^{2} \\
& +\zeta_{1, k+1} f_{1, k+1}+\zeta_{2, k+1} f_{2, k+1}+\zeta_{3, k+1} f_{3, k+1}+\zeta_{4, k+1} f_{4, k+1} \\
& +\zeta_{5, k+1} f_{5, k+1}+\zeta_{6, k+1} f_{6, k+1} A_{k} L_{k}+B_{k} H_{k}^{+}+C_{k} H_{k}^{-}+D_{k} Q_{k}^{t}+\frac{E_{k}}{2} u_{1, k}^{2}+\frac{F_{k}}{2} u_{2, k}^{2} \\
& +\frac{G_{k}}{2} u_{3, k}^{2}+\frac{M_{k}}{2} u_{4, k}^{2}+\zeta_{1, k+1}\left[\Lambda+(1-\mu) P_{k}-\alpha_{1}\left(1-u_{1, k}\right) \frac{P_{k} L_{k}}{N}-\alpha_{2}\left(1-u_{2, k}\right) \frac{P_{k} H_{k}^{-}}{N}\right] \\
& +\zeta_{2, k+1}\left[\left(1-\mu-\beta_{1}\right) L_{k}+\theta Q_{k}^{t}+\alpha_{1}\left(1-u_{k}\right) \frac{P_{k} L_{k}}{N}-\beta_{2}\left(1-u_{2, k}\right) \frac{L_{k} H_{k}^{-}}{N}\right] \\
& +\zeta_{3, k+1}\left[\left(1-\mu-\beta_{3}-\gamma\right) H_{k}^{+}+\beta_{1} L_{k}+\beta_{2}\left(1-u_{2, k}\right) \frac{L_{k} H_{k}^{-}}{N}\right] \\
& +\zeta_{3, k+1}\left[\alpha_{2}\left(1-u_{2, k}\right) \frac{P_{k} H_{k}^{-}}{N}-u_{3, k} H_{k}^{-}\right]+\zeta_{4, k+1}\left[(1-\mu-\lambda) H_{k}^{-}+\beta_{3} H_{k}^{+}\right] \\
& +\zeta_{5, k+1}\left[(1-\mu-\theta) Q_{k}^{t}+\gamma\left(1-\sigma_{1}\right) H_{k}^{+}+\lambda \sigma_{2} H_{k}^{-}-u_{4, k} Q_{k}^{t}\right] \\
& +\zeta_{6, k+1}\left[(1-\mu) Q_{k}^{p}+\lambda\left(1-\sigma_{2}\right) H_{k}^{-}+\gamma \sigma_{1} H_{k}^{+}+u_{3, k} H_{k}^{-}+u_{4, k} Q_{k}^{t}\right] .
\end{aligned}
$$

For $k=0,1, \ldots, T-1$, the optimal controls $u_{1, k}$, $u_{2, k}, u_{3, k}$, and $u_{4, k}$ can be solved from the optimality condition:

$$
\begin{gathered}
\frac{\partial H_{k}}{\partial u_{1, k}}=0, \\
\frac{\partial H_{k}}{\partial u_{2, k}}=0, \\
\frac{\partial H_{k}}{\partial u_{3, k}}=0, \\
\frac{\partial H_{k}}{\partial u_{4, k}}=0,
\end{gathered}
$$

which are

$$
\begin{aligned}
\frac{\partial H_{k}}{\partial u_{1, k}}= & E_{k} u_{1, k}-\left(\zeta_{2, k+1}-\zeta_{1, k+1}\right) \alpha_{1} \frac{P_{k} L_{k}}{N}=0, \\
\frac{\partial H_{k}}{\partial u_{2, k}}= & F_{k} u_{2, k}-\left(\zeta_{3, k+1}-\zeta_{1, k+1}\right) \alpha_{2} \frac{P_{k} H_{k}^{-}}{N} \\
& -\left(\zeta_{3, k+1}-\zeta_{2, k+1}\right) \beta_{2} \frac{L_{k} H_{k}^{-}}{N}=0, \\
\frac{\partial H_{k}}{\partial u_{3, k}}= & G_{k} u_{3, k}-\zeta_{3, k+1} H_{k}^{-}+\zeta_{6, k+1} H_{k}^{-}=0, \\
\frac{\partial H_{k}}{\partial u_{4, k}}= & M_{k} u_{4, k}-\left(\zeta_{5, k+1}-\zeta_{6, k+1}\right) Q_{k}^{t}=0 .
\end{aligned}
$$




$$
\begin{aligned}
& u_{1, k}=\frac{1}{E_{k}}\left[\left(\zeta_{2, k+1}-\zeta_{1, k+1}\right) \alpha_{1} \frac{P_{k} L_{k}}{N}\right], \\
& u_{2, k}=\frac{1}{N F_{k}}\left[\left(\zeta_{3, k+1}-\zeta_{1, k+1}\right) \alpha_{2} P_{k} H_{k}^{-}+\left(\zeta_{3, k+1}-\zeta_{2, k+1}\right) \beta_{2} L_{k} H_{k}^{-}\right], \\
& u_{3, k}=\frac{1}{G_{k}}\left[\left(\zeta_{3, k+1}-\zeta_{6, k+1}\right) H_{k}^{-}\right], \\
& u_{4, k}=\frac{1}{M_{k}}\left[\left(\zeta_{5, k+1}-\zeta_{6, k+1}\right) Q_{k}^{t}\right] .
\end{aligned}
$$

By the bounds in $U_{a d}$ of the controls, it is easy to obtain $u_{1, k}^{*}, u_{2, k}^{*}, u_{3, k}^{*}$, and $u_{4, k}^{*}$ in the form of (9).

\section{Simulation}

In this section, we present the results obtained by solving numerically the optimality system. This system consists of the state system, adjoint system, initial and final time conditions, and the control characterization.

In this formulation, there were initial conditions for the state variables and terminal conditions for the adjoints. That is, the optimality system is a two-point boundary value problem with separated boundary conditions at time steps $k=0$ and $k=T$. We solve the optimality system by an iterative method with forward solving of the state system followed by backward solving of the adjoint system. We start with an initial guess for the controls at the first iteration, and then, before the next iteration, we update the controls by using the characterization. We continue until convergence of successive iterates is achieved.

4.1. Discussion. In this section, we study and analyse numerically the effects of the optimal control strategies such as awareness programs through media and education, contact prevention through security campaigns, treatment, and psychological support along with follow-up for the drug consumers. The numerical solution of model (2) is executed using Matlab with the following parameter values and initial values of state variable in Table 1.

The proposed control strategies in this work help to achieve several objectives:

\subsubsection{Objective A: Protecting and Preventing Potential Drug} Users and the Light Drug Users from Falling into Drug Use and Addiction. Due the importance of the awareness programs through media and education in restricting the spread of drug use, we propose an optimal strategy for this purpose. Hence, we activate the optimal control variable $u_{1}$, which represents awareness programs for the light drug users. Figure 2(a) compares the evolution of the light drug users with and without control $u_{1}$ in which the effect of the proposed awareness programs through media and education is proven to be positive in decreasing the number of light drug users and preventing potential drug users from contacting light drug users (Figure 2(b)).

4.1.2. Objective B: Decreasing the Number of Heavy Drug Users and Dealers by Preventing Contact through Security Campaigns. When the number of drug users is so high, it is obligatory to resort to some strategies such as preventing contact through security campaigns in order to reduce the number of heavy drug users-dealers (Figure 3(c)); and to protect light drug users from contacting heavy drug users-dealers through security campaigns which also has a positive effect on reducing the number of the heavy drug users (Figure 3(b)). Therefore, we propose an optimal strategy by using the optimal control $u_{2}$ in the beginning. In spite of using the optimal control $u_{2}$, we observe that the number of light drug consumers increases due to protecting them from contacting the heavy drug users-dealers. The reason of this increase is justified by the fact that light drug consumers revert back to using drugs occasionally (Figure 3(a)). Also, the proposed strategy has an additional effect in decreasing clearly the number of heavy drug users and heavy drug users-dealers and providers.

\subsubsection{Objective C: Treatment in the Addiction Centers.} Given the importance and effectiveness of this strategy, we use the control strategy to encourage heavy drug users to know about treatment centers and join them to decrease the spread of drug consumers.

We propose treatment within addiction centers represented by the strategy of optimal control $u_{3}$. From Figures 4(a) and 4(b), the decrease of the number of heavy drug users is clearly achieved which in turn had a positive effect on reducing the number of heavy drug users-dealers and providers.

4.1.4. Objective D: Control with Psychological Support along with Follow-Up. Taking into consideration the importance and the effectiveness of this strategy on the individuals who temporarily quit drug, we propose an optimal strategy by using the optimal control $u_{4}$ in the beginning which represents follow-up and psychological support to prevent the temporary quitters from reverting back to using the drugs occasionally $(\theta \neq 0)$ (Figure $5(b))$. The proposed strategy has an additional effect in decreasing clearly the number of temporary quitters of drug consumption. Figure 5(a) shows that the number of light drug users is decreased markedly $(\theta=0)$.

Note: several optimal controls can be combined to achieve other objectives and implement other strategies depending on the phenomenon and the particularity of each society. 
TABLE 1: The description of parameters used for the definition of discrete time system (1). We used just arbitrary academic data.

\begin{tabular}{|c|c|c|c|c|c|c|c|c|c|c|c|c|c|c|c|c|c|}
\hline$P_{0}$ & $L_{0}$ & $H_{0}^{+}$ & $H_{0}^{-}$ & $Q_{0}^{t}$ & $Q_{0}^{p}$ & $\Lambda$ & $\alpha_{1}$ & $\alpha_{2}$ & $\mu$ & $\beta_{1}$ & $\beta_{2}$ & $\beta_{3}$ & $\gamma$ & $\lambda$ & $\sigma_{1}$ & $\sigma_{2}$ & $\theta$ \\
\hline $5.10^{3}$ & $3.10^{3}$ & $1,5.10^{3}$ & $1.10^{3}$ & $2.10^{3}$ & $1.10^{3}$ & $5.10^{2}$ & 0.45 & 0.5 & 0.04 & 0.025 & 0.2 & 0.1 & 0.05 & 0.05 & 0.05 & 0.7 & 0.05 \\
\hline
\end{tabular}

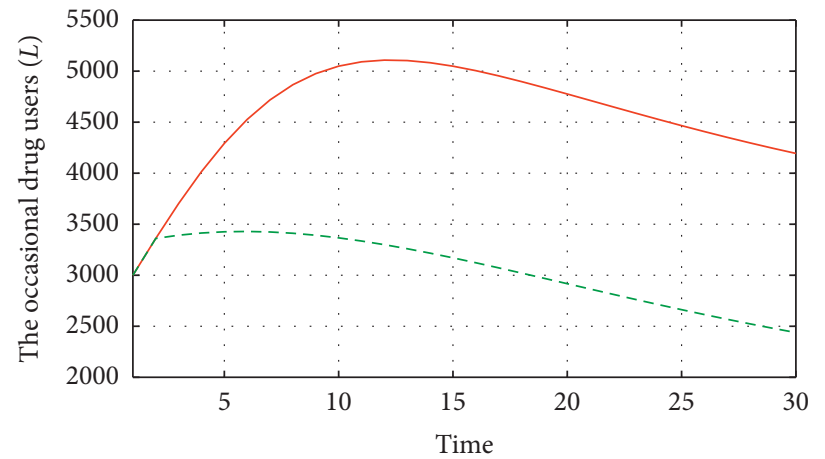

- $L$ without control

- - $L$ with control $u_{1}$

(a)

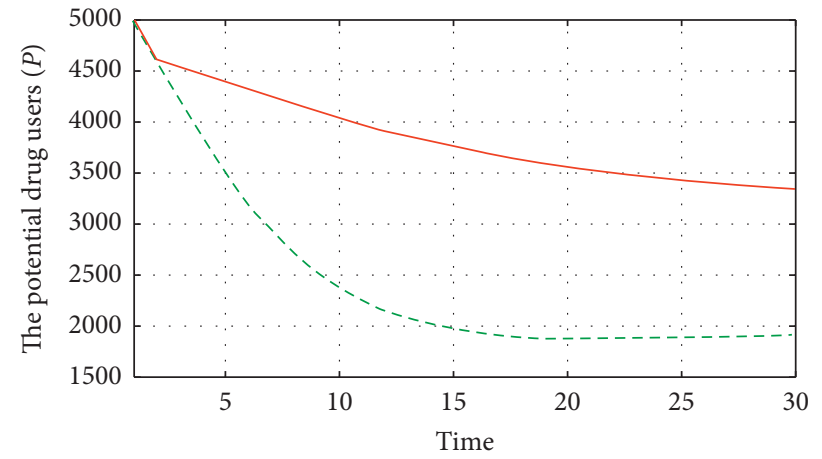

-- $P$ without control

— P with control $u_{1}$

Figure 2: (a) The evolution of the $L$ with and without controls. (b) The evolution of the P with and without controls.

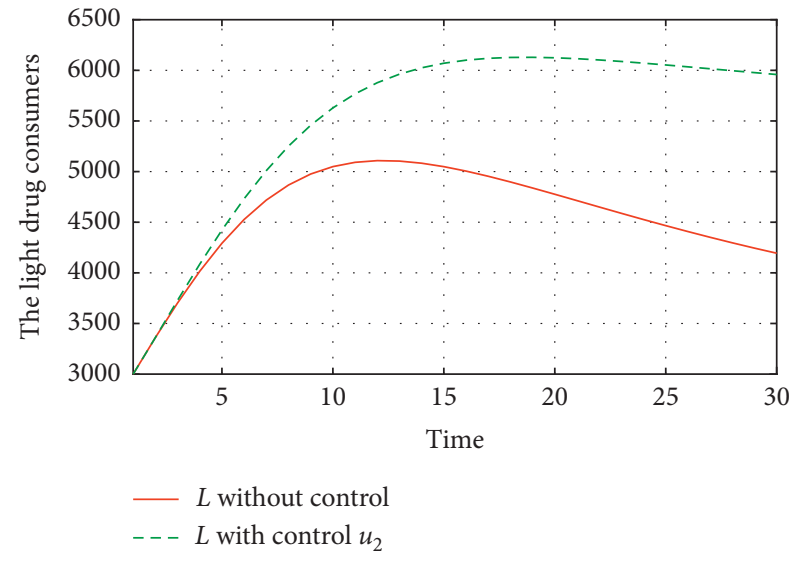

(a)

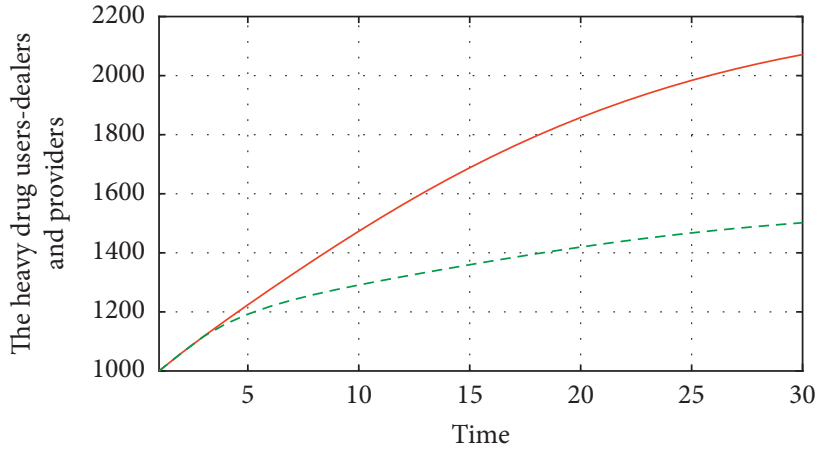

- $H^{-}$without control

--- $H^{-}$with control $u_{2}$

(b)

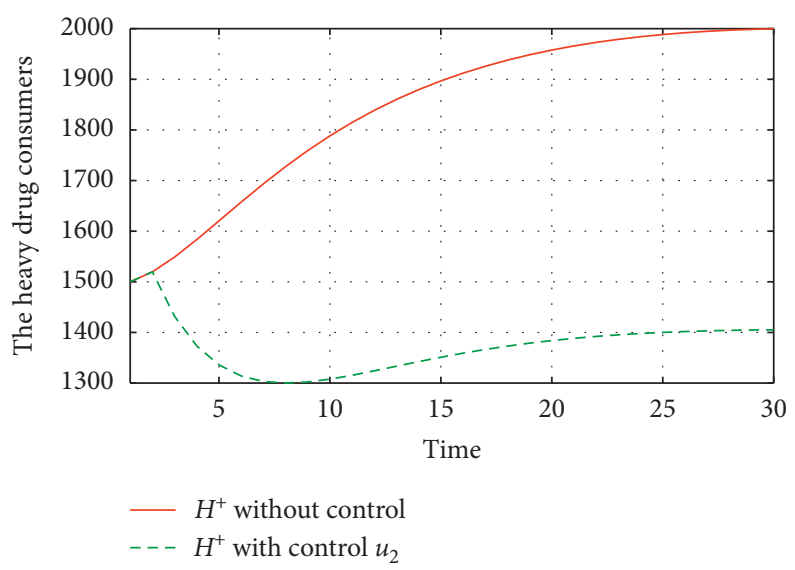

(c)

Figure 3: (a) The evolution of $L$ with and without controls. (b) The evolution of the $H^{-}$with and without controls. (c) The evolution of the $H^{+}$ with and without controls. 


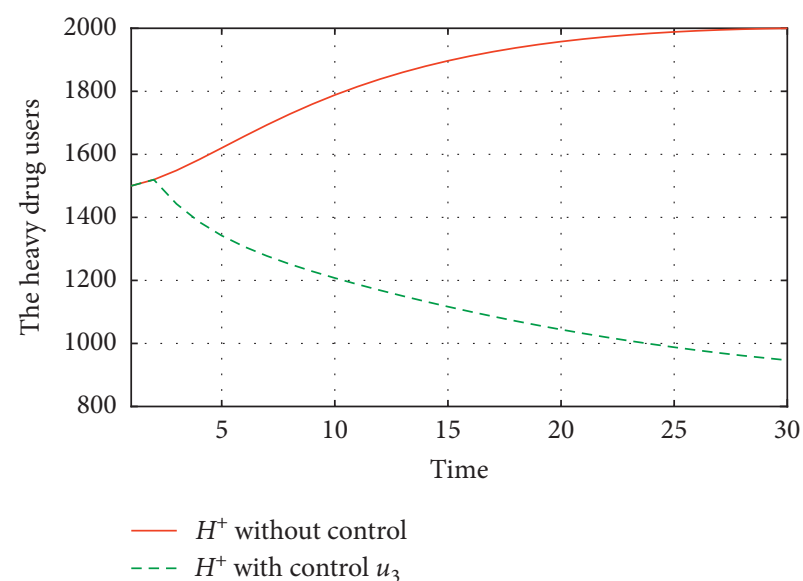

(a)

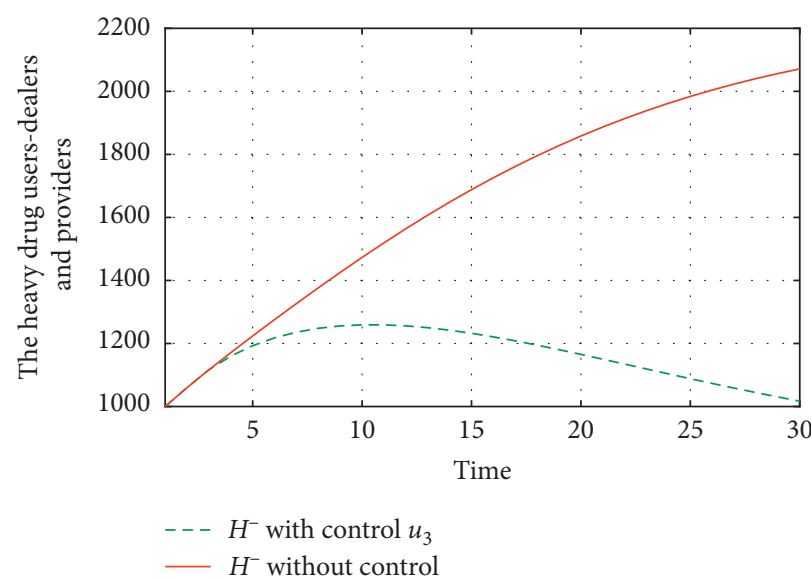

(b)

Figure 4: (a) The evolution of the $H^{+}$with and without controls. (b) The evolution of the $H^{-}$with and without controls.

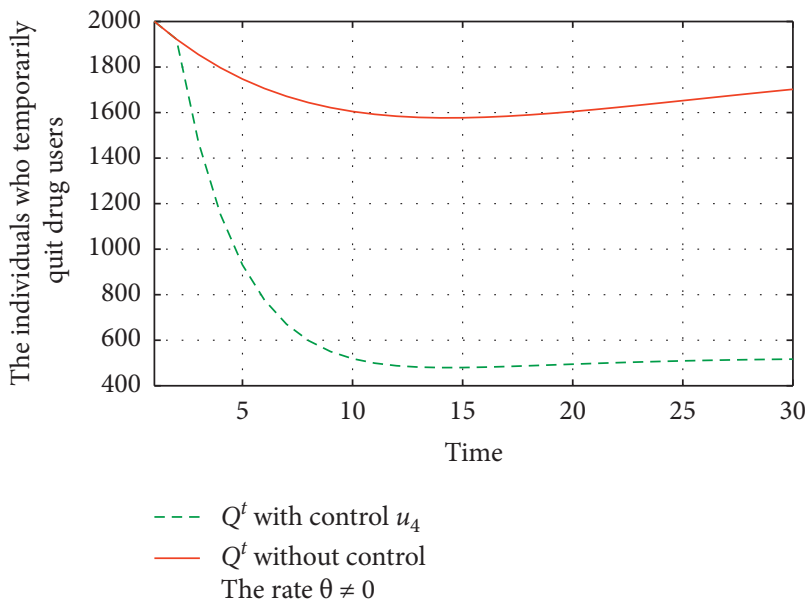

(a)

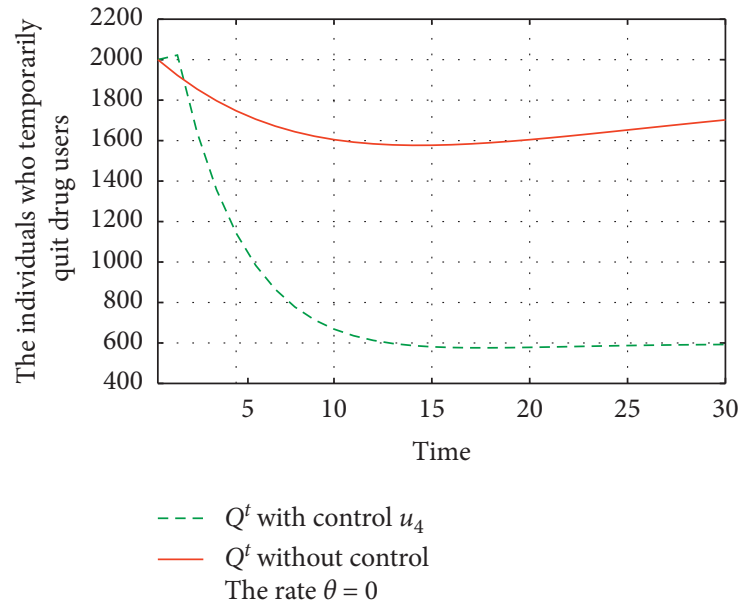

(b)

Figure 5: (a) The evolution of the $Q^{t}$ with and without controls with $\theta \neq 0$. (b) The evolution of the $Q^{t}$ with and without controls with $\theta=0$.

\section{Conclusion}

In this paper, we introduced a discrete modeling of drug users in order to minimize the number of light drug users, heavy drug users, heavy drug users-dealers, and temporary quitters of drug consumption. We also introduced four controls which, respectively, represent awareness programs through education and media, contact prevention through security campaigns, treatment, and psychological support along with follow-up. We applied the results of the control theory and we managed to obtain the characterisations of the optimal controls. The numerical simulation of the obtained results showed the effectiveness of the proposed control strategies.

\section{Data Availability}

No data were used to support this study.

\section{Disclosure}

This article was presented at the International Conference on Research in Applied Mathematics and Computer Science (ICRAMC) 2020, which took place on July 15-18, 2020.

\section{Conflicts of Interest}

The authors declare that there are no conflicts of interest regarding the publication of this paper.

\section{References}

[1] UNODC, "World drug report-united nations office on drugs and crime," 2019, https://www.unodc.org/wdr2019/prelaunch/ WDR19_Booklet_1_EXSUM.pdf. 
[2] WHO, "HIV 2016-2021-world health organization," 2016, https://apps.who.int/iris/bitstream/handle/10665/246178/WHOHIV-2016.05-eng.pdf.

[3] EMCDDA, "The European monitoring centre for drugs and drug addiction," http://www.emcdda.europa.eu/data/stats2019/gps.

[4] F. El Omari and T. Jallal, "The mediterranean school survey project on alcohol and other drugs in Morocco," Addicta: The Turkish Journal on Addictions, vol. 2, pp. 30-39, 2015.

[5] K. Bucher, "Bernadette, mathematically modeling the spread of methamphetamine use," University of Alabama Libraries, Tuscaloosa, Alabama, 2014.

[6] F. Guerrero, F.-J. Santonja, and R.-J. Villanueva, "Analysing the Spanish smoke-free legislation of 2006: a new method to quantify its impact using a dynamic model," International Journal of Drug Policy, vol. 22, no. 4, pp. 247-251, 2011.

[7] Z. Hu, Z. Teng, and H. Jiang, "Stability analysis in a class of discrete SIRS epidemic models," Nonlinear Analysis: Real World Applications, vol. 13, no. 5, pp. 2017-2033, 2012.

[8] A. Labzai, O. Balatif, and M. Rachik, "Optimal control strategy for a discrete time smoking model with specific saturated incidence rate," Discrete Dynamics in Nature and Society, vol. 2018, Article ID 5949303, 10 pages, 2018.

[9] A. Lahrouz, L. Omari, D. Kiouach, and A. Belmaâti, "Deterministic and stochastic stability of a mathematical model of smoking," Statistics \& Probability Letters, vol. 81, no. 8, pp. 1276-1284, 2011.

[10] D. C. Zhang and B. Shi, "Oscillation and global asymptotic stability in a discrete epidemic model," Journal of Mathematical Analysis and Applications, vol. 278, no. 1, pp. 194-202, 2003.

[11] J. Boscoh, H. Njagarah, and F. Nyabadza, "Modelling the role of drug barons on the prevalence of drug epidemics," Mathematical Biosciences and Engineering, vol. 10, no. 3, pp. 843-860, 2013.

[12] O. Balatif, A. Labzai, and M. Rachik, "A discrete mathematical modeling and optimal control of the electoral behavior with regard to a political party," Discrete Dynamics in Nature and Society, vol. 2018, Article ID 9649014, 14 pages, 2018.

[13] V. Guibout and A. M. Bloch, "A discrete maximum principle for solving optimal control problems," in Proceedings of the 43rd IEEE Conference on Decision and Control, vol. 2, pp. 1806-1811, Nassau, Bahamas, December 2004.

[14] D. Wandi, R. Hendon, B. Cathey, E. Lancaster, and R. Germick, "Discrete time optimal control applied to pest control problems," Involve, a Journal of Mathematics, vol. 7, no. 4, pp. 479-489, 2014.

[15] S. Mushayabasa and G. Tapedzesa, "Modeling illicit drug use dynamics and its optimal control analysis," Computational and Mathematical Methods in Medicine, vol. 2015, Article ID 383154, 11 pages, 2015.

[16] A. Zeb, G. Zaman, and S. Momani, "Square-root dynamics of a giving up smoking model," Applied Mathematical Modelling, vol. 37, no. 7, pp. 5326-5334, 2013.

[17] L. S. Pontryagin, V. G. Boltyanskii, R. V. Gamkrelidze, and E. F. Mishchenko, The Mathematical Theory of Optimal Processes, Wiley, New York, NY, USA, 1962.

[18] M. D. Rafal and W. F. Stevens, "Discrete dynamic optimization applied to on-line optimal control," AlChE Journal, vol. 14, no. 1, pp. 85-91, 1968.

[19] C. L. Hwang and L. T. Fan, "A discrete version of pontryagin's maximum principle," Operations Research, vol. 15, no. 1, pp. 139-146, 1967. 